\title{
Médiévales
}

Langues, Textes, Histoire

76 | printemps 2019

Du nouveau en archives

\section{Florian BESSON, Justine BRETON (dir.), Kaamelott, un} livre d'histoire

Paris, Vendémiaire, 2018, 336 p.

\section{Alban Gautier}

\section{(2) OpenEdition}

Journals

Édition électronique

URL : https://journals.openedition.org/medievales/10129

DOI : 10.4000/medievales. 10129

ISSN : 1777-5892

Éditeur

Presses universitaires de Vincennes

\section{Édition imprimée}

Date de publication : 5 septembre 2019

Pagination : 174-175

ISBN : 978-2-37924-028-7

ISSN : 0751-2708

Référence électronique

Alban Gautier, «Florian besson, Justine bReton (dir.), Kaamelott, un livre d'histoire », Médiévales [En ligne], 76 | printemps 2019, mis en ligne le 05 septembre 2019, consulté le 22 avril 2022. URL : http://

journals.openedition.org/medievales/10129; DOI : https://doi.org/10.4000/medievales.10129

Ce document a été généré automatiquement le 22 avril 2022.

Tous droits réservés 


\title{
Florian BESSON, Justine BRETON (dir.), Kaamelott, un livre d'histoire
}

Paris, Vendémiaire, 2018, 336 p.

\author{
Alban Gautier
}

\section{RÉFÉRENCE}

Florian BESSON, Justine BRETON (dir.), Kaamelott, un livre d'histoire, Paris, Vendémiaire, $2018,336 \mathrm{p}$.

1 La série télévisée comique Kaamelott, créée, scénarisée, réalisée et interprétée par Alexandre Astier et diffusée sur la chaîne M6 entre 2005 et 2009, est, comme le soulignent les éditeurs de cet agréable volume, " une des plus ambitieuses réécritures de la légende arthurienne : six saisons, 458 épisodes, pour un total de près de trentehuit heures » (p. 10). Les actes d'un colloque tenu à Paris en 2017 et publiés - saluons la performance - moins d'un an plus tard, nous invitent à en explorer les ressorts.

2 L'approche retenue par les auteurs des vingt contributions relève principalement des études médiévalistes. Ce livre vise dès lors deux objectifs distincts, mais complémentaires. Il s'agit en premier lieu d'éclairer le rapport des concepteurs, mais aussi des spectateurs de Kaamelott, à la matière de Bretagne et plus largement au Moyen Âge, à la fois en tant que moment du passé, en tant qu'objet d'étude des médiévistes et en tant qu'objet culturel contemporain médiatisé par de nombreux canaux. Il s'agit aussi de s'appuyer sur l'analyse de cette série pour comprendre, par comparaison avec d'autres productions culturelles actuelles, les multiples façons dont notre société perçoit, s'approprie et réinvente un Moyen Âge et une légende arthurienne à son usage. Ces deux objectifs - élucidation des ressorts médiévalistes de la série et éclairage plus vaste sur ce phénomène dans la France et l'Occident du début $\mathrm{du} \mathrm{XXI}^{\mathrm{e}}$ siècle - sont poursuivis de manière inégale par les diverses contributions. L'intérêt de chaque article est donc variable selon ce que le lecteur vient y chercher. Car le livre, écrit par des universitaires et qui répond de fait à un cahier des charges 
universitaire d'analyse historique, sémiotique, littéraire... semble cibler deux lectorats différents.

3 Les fans de la série et tous ceux qui sont curieux de comprendre ce que devient tel ou tel trait médiéval à la moulinette du médiévalisme d'Alexandre Astier trouveront évidemment leur bonheur dans les articles qui ne s'écartent qu'assez peu de la série. Leur méthodologie consiste pour l'essentiel à identifier les sources médiévales ou plus récentes (car Astier réécrit tout autant Chrétien de Troyes que T. H. White ou John Boorman) de certains traits récurrents de la série, et à montrer comment le traitement de certains personnages ou épisodes reprend des aspects du « vrai » Moyen Âge ou, au contraire, s'en démarque. Le lecteur curieux apprend ainsi comment la série traite des thèmes arthuriens classiques comme le Graal, Guenièvre et Merlin, mais aussi des mythèmes incontournables du médiévalisme comme les dragons ou la chevalerie. De façon apparemment plus anecdotique, mais souvent passionnante, il saura comment Alexandre Astier, scénariste cultivé et informé, relit la Poétique d'Aristote ou se situe par rapport aux recherches sur la musique ancienne ou sur l'histoire et la théorie des pratiques ludiques. Il comprendra aussi, dans l'intéressant article réflexif de Servane Rayne (p.67-76), comment «la Table Ronde se moque des chercheurs» et parodie ingénieusement le discours universitaire en mettant en scène une discussion pour le moins aporétique entre les chevaliers autour de la "vraie nature du Graal ", vase, pierre incandescente ou simple récipient. Mais ces articles ne sont peut-être pas ceux qui intéresseront le plus les lecteurs qui - chez les médiévistes autant que dans le grand public - ne sont pas des spectateurs fanatiques de Kaamelott et ne sont pas capables d'en réciter les moindres répliques. Pour eux, on conseillera d'abord la lecture de l'excellente introduction des deux éditeurs, qui donne en quelques pages les principales clés pour comprendre la série et la situer dans les traditions médiévales et modernes de la réécriture arthurienne.

D'autres chapitres en revanche, principalement regroupés en fin de volume, vont bien au-delà de l'analyse des épisodes : leur approche tend alors à éclairer plus largement le rapport de notre société au Moyen Âge et à Rome. Les contributions de Pauline Ducret, de Magali Coumert et Bruno Dumézil explorent nos perceptions contrastées de l'Antiquité et du Moyen Âge, et plus précisément de Rome et des barbares. Alexandre Astier a choisi de situer sa série dans une temporalité de l'entre-deux, aux environs de 500 et donc à mi-chemin de Rome et du Moyen Âge : sa Bretagne médiévale répond sans cesse à une Rome qui, dans la sixième saison de la série, est en outre filmée dans les splendides décors de Cinecittà, où a été tournée la série Rome (qui se déroule au $\mathrm{I}^{\mathrm{er}}$ siècle avant notre ère). Pour être bien compris, le médiévalisme de Kaamelott doit donc être articulé avec un "antiquitisme» (pour user d'un barbarisme désignant le rapport à l'Antiquité) : les deux rapports au passé fonctionnent ainsi en regard l'un de l'autre. L'article de Rudi Beaulant sur la justice, celui de William Blanc sur l'alimentation et celui de Pierre-Brice Stahl sur le casque des vikings s'attaquent à trois mythèmes du médiévalisme. Tout en montrant comment Alexandre Astier en use et en joue, ils offrent une réflexion sur ce à quoi le spectateur contemporain reconnaît le Moyen Âge, bien souvent à rebours des résultats auxquels sont parvenus les médiévistes. Ainsi, la justice médiévale serait toujours brutale et expéditive, le banquet médiéval verrait dominer la profusion, la viande et la graisse, et le viking serait reconnaissable à son casque à cornes. On regrettera peut-être l'absence d'un article de synthèse sur la façon dont la série et, plus largement, les visions médiévalistes contemporaines représentent le christianisme « médiéval ». Le thème est effleuré à plusieurs reprises - en particulier 
par Justine Breton dans son chapitre sur Merlin -, mais il aurait mérité un traitement spécifique.

L'article que nous avons lu toutefois avec le plus grand intérêt est le dernier des vingt ; il précède avec bonheur la conclusion, car il résume et resitue plusieurs des éléments analysés au fil des pages. Dans un chapitre intitulé «Kaamelott et les mondes de la fantasy » (p. 267-276), David Peyron s'interroge sur le statut de série culte de Kaamelott, et montre que celui-ci est étroitement articulé à la place que tient le médiévalisme dans les cultures geek contemporaines, dans lesquelles les codes de la fantasy occupent une place considérable. Faisant très souvent appel à des références relevant de ces univers culturels, la série (re)crée un monde où Alexandre Astier joue avec jubilation et où il invite le spectateur à jouer. Cette inscription d'une production télévisée française au cœur des cultures geek et des codes de la fantasy « explique la popularité de cette série auprès d'une population plutôt consommatrice de produits américains ou japonais » (p. 276). C'est en cela que l'analyse de Kaamelott et de son rapport au Moyen Âge, conduite avec talent tout au long de ce livre, éclaire si bien la façon dont tant de jeunes adultes des années 2000 et 2010 perçoivent « le médiéval».

\section{AUTEURS}

\section{ALBAN GAUTIER}

Université de Caen Normandie 